\title{
Increased levels of calcitonin gene-related peptide in serum accelerate fracture healing following traumatic brain injury
}

\author{
YANG SONG ${ }^{*}$, LONG BI ${ }^{*}$, ZHENYU ZHANG, ZHAOSONG HUANG, \\ WEI HOU, XU LU, PENGXIAO SUN and YISHENG HAN
}

\author{
Department of Orthopedics, Xijing Hospital, the Fourth Military Medical University, Xi'an 710032, P.R. China
}

Received July 2, 2011; Accepted October 19, 2011

DOI: $10.3892 / \mathrm{mmr} .2011 .645$

\begin{abstract}
The mechanisms responsible for the phenomenon of an accelerated speed of fracture healing in patients with traumatic brain injury (TBI) remain unclear. The present study was performed to test the hypothesis that TBI causes changes in calcitonin gene-related peptide (CGRP) levels in sera that enhance fracture healing. A standard closed femoral fracture was produced in rats, which were subjected to additional closed head trauma. The fracture healing was assessed 4 and 8 weeks later using micro-CT. Sera, brain tissues and muscles surrounding the fracture sites collected at 24, 48, 72 and $168 \mathrm{~h}$ after injury were used to detect the expression of CGRP using ELISA, immunohistochemistry and RT-PCR. Micro-CT demonstrated that fracture healing and mineralization in the TBI-fracture group occurred earlier compared to the fracture-only group. ELISA analysis revealed a high concentration of CGRP in the TBI-fracture group $(\mathrm{P}<0.05)$, and immunohistochemistry assay and RT-PCR analysis revealed a significant increase in CGRP in the brain and muscle of the TBI-fracture group at $168 \mathrm{~h}$ after fracture $(\mathrm{P}<0.001)$. Our results indicate that the mechanism for the enhancement of fracture-healing secondary to traumatic brain injury is correlated to the high levels of CGRP, which may be released from the brain tissue into the serum.
\end{abstract}

\section{Introduction}

In our clinical studies, we noted an accelerated speed of fracture-healing in patients with traumatic brain injury (TBI). In 1987, Perkins and Skirving (1) demonstrated increased callus formation and a shorter time to union in patients with long-bone fractures and TBI. However, the mechanisms underlying this phenomenon are still unknown. During subse-

Correspondence to: Dr Yisheng Han, Department of Orthopedics, Xijing Hospital, the Fourth Military Medical University, Xi'an 710032, P.R. China

E-mail: yishenghan@hotmail.com

*Contributed equally

Key words: calcitonin gene-related peptide, traumatic brain injury, fracture healing quent decades, a series of studies were carried out to attempt to identify the mechanism responsible for this phenomenon. Bidner et al (2) provided clear evidence that patients with TBI possess a humoral mechanism for enhanced fracture healing and that TBI is associated with an increased amount of certain stimulating factors found in serum. Calcitonin gene-related peptide (CGRP) is among these factors and may induce enhanced osteogenesis in patients with TBI.

CGRP is a 37-amino acid neuropeptide encoded by the calcitonin gene. CGRP has an osteogenic stimulating effect by stimulating stem cell mitosis and/or osteoprogenitor cell differentiation in vitro (3). CGRP is also capable of inhibiting bone resorption in vitro and inducing hypocalcemia in vivo (4). Moreover, during hematencephalon formation, the secretion of CGRP markedly increases (5), indicating that CGRP may be released directly by the injured brain tissue. The present study aimed to determine whether CGRP is one of the unidentified humoral factors that cause enhanced osteogenesis in patients with TBI and to identify the downstream pathway that is activated by increased CGRP expression.

\section{Materials and methods}

Animals. Male Sprague-Dawley rats (Laboratory Animal Center of the Fourth Military Medical University, Xi'an, China) weighing between 300 and $350 \mathrm{~g}$ were used for the experiments. The rats were randomly divided into four groups: TBI-fracture, fracture-only, TBI-only and control (the numbers of experimental animals in each group at each time point are shown in Table I). Animal experiments were conducted according to the Chinese Regulations of Animal Welfare and permission was granted by the Ethical Committee of the Fourth Military Medical University.

Surgical technique. Closed, transverse femoral shaft fractures were produced as described by Bonnarens and Einhorn (6). Briefly, animals were weighed on a scale and anesthetized with an intraperitoneal injection of $20 \%$ urethane solution ( $5 \mathrm{ml} / \mathrm{kg}$ of body weight). The left hind limb was shaved, disinfected and draped. A median parapatellar incision was made, followed by a median parapatellar incision into the joint capsule extending from the midline through the vastus medialis muscle to the patellar tendon insertion. Slow flexion of the knee and movement of the patella achieved lateral dislo- 
Table I. Animal numbers at each time point and various measurements.

\begin{tabular}{|c|c|c|c|c|c|c|c|c|c|c|c|c|c|}
\hline \multirow[t]{2}{*}{ Group } & \multicolumn{2}{|c|}{$\begin{array}{l}\text { Micro-CT } \\
\text { analysis }\end{array}$} & \multicolumn{3}{|c|}{$\begin{array}{l}\text { Immunohistochemistry } \\
\text { assay }\end{array}$} & \multicolumn{3}{|c|}{$\begin{array}{l}\text { RT-PCR } \\
\text { analysis }\end{array}$} & \multicolumn{5}{|c|}{ ELISA analysis } \\
\hline & $4 \mathrm{~W}$ & $8 \mathrm{~W}$ & $0 \mathrm{~h}$ & $72 \mathrm{~h}$ & $168 \mathrm{~h}$ & $0 \mathrm{~h}$ & $72 \mathrm{~h}$ & $168 \mathrm{~h}$ & $0 \mathrm{~h}$ & $24 \mathrm{~h}$ & $48 \mathrm{~h}$ & $72 \mathrm{~h}$ & $168 \mathrm{~h}$ \\
\hline TBI-fracture & 6 & 6 & 0 & 6 & 6 & 0 & 6 & 6 & 0 & 6 & 6 & 6 & 6 \\
\hline Fracture-only & 6 & 6 & 0 & 6 & 6 & 0 & 6 & 6 & 0 & 6 & 6 & 6 & 6 \\
\hline TBI-only & 0 & 0 & 0 & 6 & 6 & 0 & 0 & 0 & 0 & 6 & 6 & 6 & 6 \\
\hline Control & 6 & 0 & 6 & 0 & 0 & 6 & 0 & 0 & 6 & 0 & 0 & 0 & 0 \\
\hline
\end{tabular}

cation. An intercondylar entry point for the insertion of the 1.2-mm diameter Kirschner wire into the medullary space was performed with a handheld drill. The wire was inserted until it touched the greater trochanter. The wire was then retracted slightly, cut, reinserted, and buried under the cartilage surface of the knee. The operative site and the skin were closed with sutures. A fracture was then produced with a blunt guillotine, and radiographs were generated to confirm the pin placement and fracture configuration. Fractures that did not occur in the mid-part of the diaphysis or comminuted were excluded.

Traumatic brain injury. Traumatic brain injury (TBI) was generated through the application of a well-established impact acceleration system $30 \mathrm{~min}$ after fracture $(7,8)$. This model has been shown to reproducibly impart a diffuse axonal injury similar to that observed in patients with a TBI. When the animal showed signs of normal recovery from the closed femoral shaft fracture, a nickel-plated cap was fitted to the top of the animal's skull. The animal's head was then positioned on a foam block under stainless steel tubing with a smooth inner wall. The opening of the tubing was centered over the nickel plate directly above the animal's head. A 250-g weight was then dropped from a height of exactly $1 \mathrm{~m}$. Once impact was made, the foam block and the animal were moved away from the tubing to avoid a rebound impact, thus limiting the injury to a single impaction. Animals were returned to their cages and allowed to recover under observation.

Specimen collection. Blood samples were collected from rats in the TBI-fracture, fracture-only and TBI-only groups at 4 time points during the first week $(24,48,72$ and $168 \mathrm{~h}$ after injury) and from the control group at $24 \mathrm{~h}$ prior to injury. Samples were centrifuged at $1000 \mathrm{x}$ g for $15 \mathrm{~min}$ within 30 min of collection, and the resulting serum was stored at $-80^{\circ} \mathrm{C}$ for enzyme-linked immunosorbent assay (ELISA) analysis. Whole brain tissues were harvested at 72 and $168 \mathrm{~h}$ after injury, formalin-fixed, and mounted for analysis with immuno-histochemistry. Muscles surrounding the fracture site were also dissected 72 and $168 \mathrm{~h}$ after injury, and total RNA was isolated and immediately stored at $-80^{\circ} \mathrm{C}$ for analysis with real-time polymerase chain reaction (RT-PCR). Fractured limbs were collected at 4 and 8 weeks after injury for the micro-CT scan. Blood samples, brain, muscles, and bones were also obtained from the control group. The specimens were obtained at approximately 8:00 a.m. to maintain similar conditions for all animal groups.
Micro-CT analysis. Bone specimens were scanned using the eXplore Locus micro-CT scanner (GE Healthcare, Piscataway, NJ, USA). Specimens were placed in phantom $(44 \mathrm{ml})$ and scanned perpendicular to the long bone axis at a tube voltage of $80 \mathrm{kV}$ and a tube current of $80 \mathrm{~mA}$. The region of interest for each animal tissue defined the fracture line as the center and the upper and lower $4 \mathrm{~mm}$ as two margins. Bone analysis was conducted using Microview software ABA version 2.1.2 (GE Health Care). For femoral analysis, bone mineral density (BMD) and bone mineral content (BMC) were measured in newly-formed bones. Data analysis and calculations were automatically performed by the software.

Immunohistochemistry assay. A $4-\mu \mathrm{m}$ tissue section was prepared on a slide. After the slides were deparaffinized and dehydrated using xylene and ethanol, respectively, the slides underwent antigen retrieval with a 10-min microwave pretreatment. Endogenous peroxidase in the tissue was blocked by incubating the slides with $3 \% \mathrm{H}_{2} \mathrm{O}_{2}$. After washing with phosphate-buffered saline (PBS), slides were incubated overnight with a rabbit anti-rat CGRP primary polyclonal antibody (Abcam, Cambridge, UK) at $4^{\circ} \mathrm{C}$. Slides were then rinsed and treated for $15 \mathrm{~min}$ with a secondary goat anti-rabbit antibody (Abcam) followed by treatment with an avidinbiotin reagent for $30 \mathrm{~min}$, incubated in diaminobenzidine for $10 \mathrm{~min}$, counterstained with hematoxylin, dehydrated in a graded series of alcohols, cleared in xylene and mounted. Sections from normal brain samples were obtained from the control group.

Reverse transcription of $m R N A$ and RT-PCR analysis. Total RNA was isolated from muscles using TRIzol (Invitrogen, Carlsbad, CA, USA) according to the manufacturer's instructions. The primer sequences used for CGRP were 5'-AAGTTC TCCCCTTTCCTG-3' (sense) and 5'-GCCTCCTGTTCC TCC TC-3' (antisense). The PCR protocol consisted of 1 cycle at $94^{\circ} \mathrm{C}$ for $30 \mathrm{sec}, 30$ cycles at $52^{\circ} \mathrm{C}$ for $30 \mathrm{sec}, 1 \mathrm{cycle}$ at $72^{\circ} \mathrm{C}$ for $30 \mathrm{sec}$, and 1 cycle at $72^{\circ} \mathrm{C}$ for $8 \mathrm{~min}$. $\beta$-actin was amplified as the internal marker, and the primer sequences used for $\beta$-actin were 5'-TGCCGCATCCTCTTCCT-3 (sense) and 5'-GGA TGTCAACGTCACACTTC-3' (antisense). The ratio of CGRP mRNA to $\beta$-actin mRNA was calculated by average light density analysis to determine the level of CGRP mRNA expression in the muscles surrounding the fracture. Each molecular test was repeated 3 times and the control was used in the molecular testing. 
Table II. Micro-CT data.

\begin{tabular}{|c|c|c|c|c|}
\hline \multirow[t]{2}{*}{ Group } & \multicolumn{2}{|c|}{ BMC (mg) } & \multicolumn{2}{|c|}{ BMD (mg/cc) } \\
\hline & $4 \mathrm{~W}$ & $8 \mathrm{~W}$ & $4 \mathrm{~W}$ & $8 \mathrm{~W}$ \\
\hline TBI-fracture & $0.9150 \pm 0.1070$ & $0.3575 \pm 0.056$ & $599.1258 \pm 41.6590$ & $727.0774 \pm 56.3459$ \\
\hline Fracture-only & $0.5934 \pm 0.1057$ & $0.5142 \pm 0.0794$ & $526.5637 \pm 43.3673$ & $736.2044 \pm 66.9357$ \\
\hline
\end{tabular}

BMC, bone mineral content; BMD, bone mineral density.
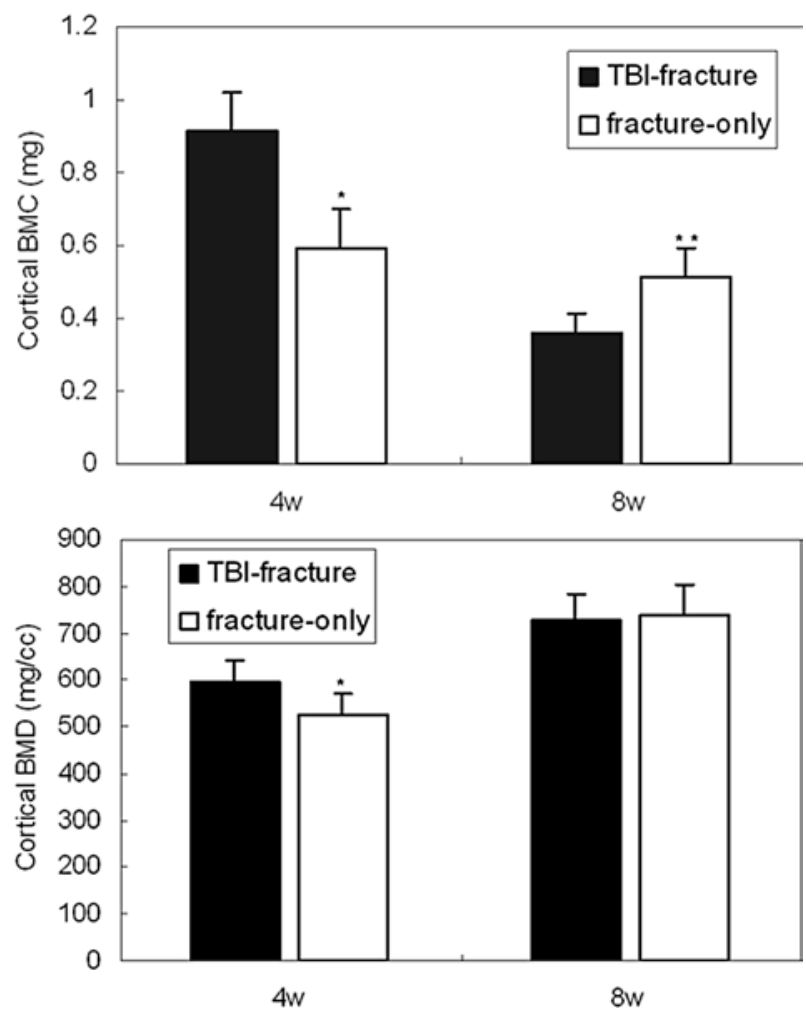

Figure 1. Micro-CT examination of newly-formed bones in the TBI-fracture and fracture-only groups at various time points post-injury (4 and 8 weeks). Calculations for the indicated parameters were performed using Microview ABA 2.1.2 software. Results are the mean \pm SD of values from 5 male rats. ${ }^{*} \mathrm{P}<0.01,{ }^{* *} \mathrm{P}<0.05$. BMC, bone mineral content; BMD, bone mineral density.

ELISA analysis. The CGRP ELISA immunoassay kit (SPI-Bio, Montigny-le-Bretonneux, France) was used to quantify the amount of rat CGRP present in the serum. The CGRP assay was performed according to the manufacturer's instructions. The optical density was measured at a wavelength of $450 \mathrm{~nm}$ using an ELISA microplate reader (Multiskan Ascent, Thermo Labsystems, Altrincham, UK). Concentrations were calculated and are expressed as $\mathrm{pg} / \mathrm{ml}$.

Statistical analysis. Data were presented as the means \pm standard deviation (SD). The effectiveness of the treatments was analyzed by one-way analysis of variance. The Student-Newman-Keuls test was performed for multiple comparisons. Statistical analyses were carried out using the Statistical Program for Social Sciences (SPSS) version 17.0. $\mathrm{P}<0.05$ was considered to be statistically significant.

\section{Results}

General observation. Following surgery,2 ratsin the TBI-fracture group died due to head trauma, causing a substantial increase in morbidity. In each of the TBI-fracture and fracture-only groups, 1 animal was excluded due to a comminuted fracture. A total of 4 animals were excluded in our study, and were replaced. There were no signs of infection in any group and no non-unions in the TBI-fracture and fracture-only groups. The brain-injured animals exhibited clear signs of neurologic impairment at the time of injury and histological changes in the brain specimens (data not shown) (9). Similar results were previously reported in studies measuring the effectiveness of the head injury model $(7,8)$. None of the groups exhibited any difference in the ability to walk 1 day after the injury.

Micro-CT analysis. Micro-CT examination revealed that TBI enhanced bone healing following femoral shaft fractures (Fig. 1, Table II). The BMD of newly-formed bone was $599.1258 \pm 41.6590 \mathrm{mg} / \mathrm{cc}$ at 4 weeks after injury in the TBI-fracture group, $526.5637 \pm 43.3673 \mathrm{mg} / \mathrm{cc}$ at 4 weeks after injury in the fracture-only group, $727.0774 \pm 56.3459 \mathrm{mg} / \mathrm{cc}$ at 8 weeks after injury in the TBI-fracture group and $736.2044 \pm 66.9357 \mathrm{mg} / \mathrm{cc}$ at 8 weeks after injury in the fracture-only group. The BMC of newly-formed bone was $0.9150 \pm 0.1070 \mathrm{mg}$ at 4 weeks after injury in the TBI-fracture group, $0.5934 \pm 0.1057 \mathrm{mg}$ at 4 weeks after injury in the fracture-only group, $0.3575 \pm 0.0560 \mathrm{mg}$ at 8 weeks after injury in the TBI-fracture group and $0.5142 \pm 0.0794 \mathrm{mg}$ at 8 weeks after injury in the fracture-only group. In the 4th week, BMD and BMC in the TBI-fracture group were significantly greater than those in the fracture-only group, with P-values $<0.05$ and 0.01 , respectively. These results indicate that fracture-healing and mineralization in the TBI-fracture group occurred earlier than that in the fracture-only group. In the 8th week, although the BMD in the two groups exhibited no significant difference $(\mathrm{P}>0.05)$, the $\mathrm{BMC}$ in the TBI-fracture group was significantly lower than that in the fracture-only group $(\mathrm{P}<0.01)$.

Immunohistochemistry assay. CGRP was only detected in the brain of animals from the TBI-fracture and TBI-only groups at $168 \mathrm{~h}$ after fracture and was not detected at $72 \mathrm{~h}$ after fracture in the TBI-fracture and fracture-only groups. In addition, CGRP was not detected in the fracture-only group at $168 \mathrm{~h}$ after fracture, nor was it detected in the control group (Fig. 2).

RT-PCR analysis. RT-PCR revealed that the mRNA expression of CGRP increased in the TBI-fracture and fracture-only 

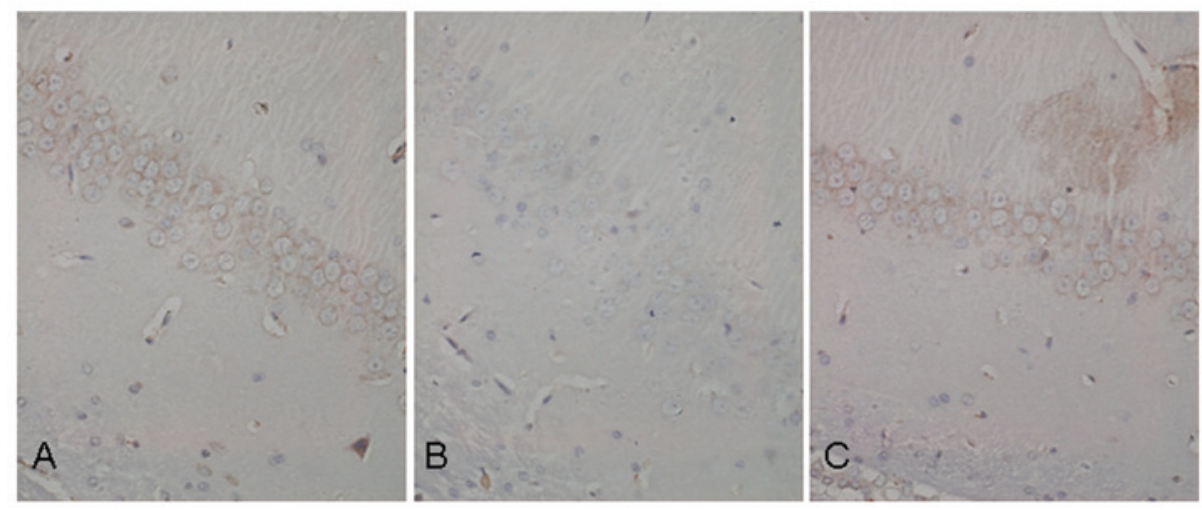

Figure 2. Expression of CGRP in the coronal section of the middle part of the brain at $168 \mathrm{~h}$ after injury (original magnification, $\mathrm{x} 400$ ). (A) TBI-fracture, (B) Fracture-only and (C) TBI-only groups. CGRP was detected in the TBI-fracture and TBI-only groups but not in the fracture-only group.

Table III. RT-PCR analysis of CGRP in muscle.

\begin{tabular}{lccc}
\hline & \multicolumn{3}{c}{ CGRP/ $\beta$-actin } \\
\cline { 2 - 4 } & $0 \mathrm{~h}$ & $72 \mathrm{~h}$ & $168 \mathrm{~h}$ \\
\hline $\begin{array}{l}\text { TBI- } \\
\text { fracture }\end{array}$ & - & $0.2528 \pm 0.0208$ & $0.3463 \pm 0.0318$ \\
Fracture- & - & $0.2644 \pm 0.0132$ & $0.2762 \pm 0.0244$ \\
only & & & - \\
Control & $0.2051 \pm 0.0168$ & - & - \\
\hline
\end{tabular}

groups at $72 \mathrm{~h}$ after injury, with P-values $<0.05$ and 0.001 when compared to the control group, respectively. At $168 \mathrm{~h}$ after injury, the level of CGRP expression in the fracture-only group was almost equal to that at $72 \mathrm{~h}(\mathrm{P}>0.05)$, whereas the CGRP expression level in the TBI-fracture group continued to increase $(\mathrm{P}<0.001)$, with a markedly higher expression when compared to the fracture-only group $(\mathrm{P}<0.01$, Fig. 3 , Table III).

ELISA analysis. The amount of rat CGRP present in the serum was tested using ELISA (Fig. 4, Table IV). At $24 \mathrm{~h}$ after injury, no apparent difference was evident in the CGRP concentration in the fracture-only and TBI-only groups compared to the control group $(\mathrm{P}>0.05)$. However, we detected more CGRP in the TBI-fracture group than in the control group $(\mathrm{P}<0.05)$. At $48 \mathrm{~h}$ after injury, the TBI-fracture, fracture-only and TBI-only groups also exhibited no difference in CGRP concentration compared to the control group $(\mathrm{P}>0.05)$. At $72 \mathrm{~h}$ after injury, the three experimental groups demonstrated higher concentrations of CGRP than the control group, and we detected higher CGRP levels in the TBI-fracture group than in the fracture-only group $(\mathrm{P}<0.05)$. At $168 \mathrm{~h}$ after injury, CGRP expression in the TBI-fracture and fracture-only groups remained constant $(\mathrm{P}>0.05)$, whereas the level of CGRP expression in the TBI-fracture group was still higher than that in the fracture-only group $(\mathrm{P}<0.05)$.

\section{Discussion}

The association between TBI and enhanced osteogenesis has been debated for many years. Clinical observations of TBI

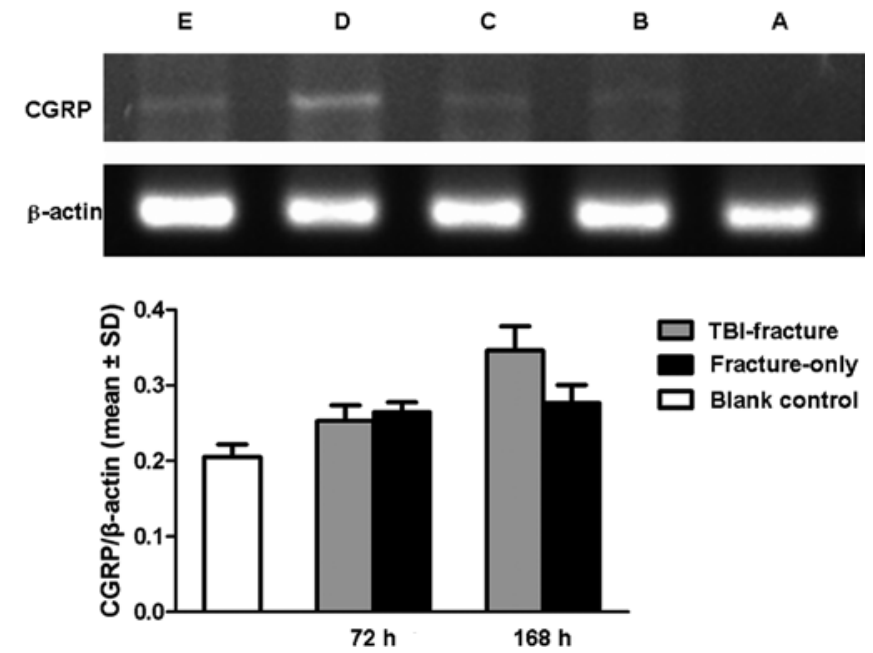

Figure 3. Expression of CGRP mRNA in different groups at various time points post-injury $(0,72$ and $168 \mathrm{~h})$. Data were calculated as a ratio of $\beta$-actin. (A) Control group $(0.2051 \pm 0.0168)$. (B) TBI-fracture group at $72 \mathrm{~h}(0.2528 \pm 0.0208)$. (C) Fracture-only group at $72 \mathrm{~h}(0.2644 \pm 0.0132)$. (D) TBI-fracture group at $168 \mathrm{~h}(0.3463 \pm 0.0318)$. (E) Fracture-only group at $168 \mathrm{~h}(0.2762 \pm 0.0244)$

patients with associated long-bone fractures have revealed abundant callus formation and a more rapid healing speed. Our micro-CT results also support findings from previous studies $(1,2)$, indicating that TBI accelerates fracture-healing time. Our data showed a significant increase in callus BMD and BMC in the TBI-fracture group as compared to the fracture-only group 4 weeks following operation. As BMD and BMC are properties of mineralization, this finding is consistent with the type of fracture-healing enhancement anticipated at an early time-point of 4 weeks. Other studies correlating callus tissue properties to fracture-healing have also demonstrated that the BMD and BMC of fracture calluses are associated with progressive changes in the material properties of the tissue since hypertrophic cartilage is mineralized and replaced with primary bone.

The observation of a smaller BMD and BMC at 8 weeks in the TBI-fracture group as compared to the fracture-only group was unexpected, and without any evidence to explain this phenomenon we can only hypothesize regarding the mechanism responsible for the difference. The reduced callus size appears 
Table IV. ELISA analysis of serum CGRP.

\begin{tabular}{lccccc}
\hline & \multicolumn{5}{c}{ CGRP $(\mathrm{pg} / \mathrm{ml})$} \\
\cline { 2 - 5 } Group & $0 \mathrm{~h}$ & $24 \mathrm{~h}$ & $48 \mathrm{~h}$ & $72 \mathrm{~h}$ & $168 \mathrm{~h}$ \\
\hline TBI-fracture & - & $27.22 \pm 10.69$ & $31.00 \pm 6.36$ & $35.33 \pm 4.81$ & $34.45 \pm 6.40$ \\
Fracture-only & - & $21.22 \pm 2.36$ & $33.89 \pm 8.89$ & $27.89 \pm 5.83$ & $27.33 \pm 4.39$ \\
TBI-only & - & $22.33 \pm 5.71$ & $18.34 \pm 3.04$ & $29.44 \pm 6.17$ & $24.00 \pm 3.44$ \\
Control & $19.22 \pm 4.52$ & - & - & - & - \\
\hline
\end{tabular}

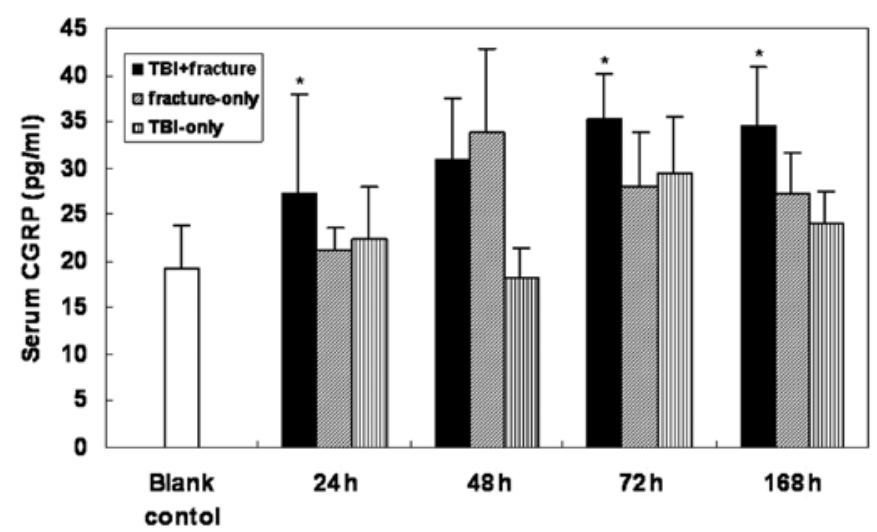

Figure 4. The concentration of CGRP in the serum of different groups at various time points post-injury $(24,48,72$ and $168 \mathrm{~h})$.

to contradict the hypothesis that TBI increases bone formation and fracture-healing. Alternatively, at an earlier stage of 4-8 weeks, the fractures in the TBI-fracture group had already progressed into the remodeling phase of fracture repair, and at 8 weeks the remodeling was almost completed. Smaller calluses, combined with the lower BMD and BMC in the TBI-fracture group, indicate that the fracture-healing and mineralization of newly-formed calluses in the TBI-fracture group occurred earlier and faster than in the fracture-only group. Boes et al identified a similar phenomenon to that observed in the present study (10). Using mechanical testing, these authors revealed that shortly ( 3 weeks) after TBI-fracture, although the fracture callus in the TBI animals was significantly stiffer than that in the fracture-only animals, there was no significant difference in torsional strength between the two groups. Moreover, the mean fracture callus diameter was significantly reduced in the TBI-fracture group compared with that in the fracture-only group. These authors also proposed that the smaller calluses in the animals with TBI may indicate that the fractures had already progressed into the remodeling phase of fracture repair. Additionally, they proposed that the lack of significant differences in the strength measurements between the TBI-fracture and fracture-only groups may indicate that the synergistic effect between TBI and fracture leads to more rapid load-transfer abilities and an acceleration of healing but only a small increase in load-carrying capacity (strength) compared with that in the fracture-only group. No effects on resorption in the normal bone (control group) were noted following the use of micro-CT in the present study. Although micro-CT was not performed in the TBI-only group, we did not observe any changes in the non-fracture area of the TBI-fracture group compared with the control group.

It is well established that numerous growth factors, hormones or peptides are transported to target tissues or organs through the serum. Whether a humoral factor is released from the injured brain or whether a direct nervous action induces enhanced fracture-healing has been debated. Of these two possibilities, we prefer the former, as the injured brain is far from the local fracture site and is not capable of acting directly upon it. Boes et al (10) revealed that in vitro human stromal stem cells proliferate beyond expected parameters when incubated with serum from rats following brain injury, and concluded that brain-derived factors mediate a mitogenic effect on osteoprogenitor cells by means of the humoral circulation. Gautschi et al (11) revealed increased proliferation of osteoblastic cells and accelerated differentiation of osteoprogenitor cells using serum from TBI and fracture patients, which also strongly supports the use of a humoral mechanism in enhanced fracture-healing and the induction of heterotopic ossification following TBI. In a previous study, Gautschi et al revealed the osteoinductive potential of cerebrospinal fluid derived from TBI patients (12). In our results, CGRP staining was observed in the choroid plexus in the TBI-fracture group but not in the fracture-only and control groups. Central nervous system-specific markers have been identified in cerebrospinal fluid and serum from brain-injured patients (13). This observation supports the hypothesis that the osteoinductive effect may reflect a disruption of the blood-brain barrier due to the trauma, allowing the leakage of osteoinductive cerebrospinal fluid components into the systemic circulation (14). Therefore, based on our data, we propose that the majority of newly formed CGRP from the damaged brain tissue flows into the serum and causes dynamic changes in serum CGRP levels. However, we cannot exclude their combined action and the possibility remains that a combined action exists.

CGRP is a neuropeptide that consists of 37 amino acid residues (15) and is generated by the alternative splicing of the Calca gene in cells of the central and peripheral nervous system (16). Numerous studies confirm the involvement of CGRP in the bone metabolism and pathophysiology of brain damage $(4,5)$. Osteoblastic cells are equipped with receptors of CGRP, and cyclic adenosine monophosphate (cAMP) production in human osteoblastic cells can be enhanced by CGRP by as much as 30-50-fold (17), leading to changes in cell morphology and function. CGRP is capable of stimulating the production of 
insulin-like growth factor-I in vitro (18) and inhibiting tumor necrosis factor (19), a cytokine that is a potent stimulator of bone resorption and an inhibitor of bone formation (20). The number of CGRP-stained fibers has been shown to increase during fracture-healing (21). Following hematencephalon formation, the secretion of CGRP clearly increases (5). In our experiment, CGRP staining in the brain was observed in the TBI-fracture group but not in the fracture-only group. CGRP expression around the fracture site markedly increased in the two groups following injury (on the third day) and continued to increase in the TBI-fracture group, whereas CGRP expression in the fracture-only group appeared to be maintained at the baseline level. Dynamic changes in CGRP serum expression were similar to those detected at the fracture site. Based on the total data in Table IV, we propose that the association between fracture healing, brain injury and CGRP levels, although the CGRP levels in the TBI-only group were not as high as those in the TBI-fracture group, they were not mostly lowest in the four groups. The TBI-only group exhibited the lowest CGRP level out of the four groups only at $48 \mathrm{~h}$ (the level was somewhat lower than that in the control group, $\mathrm{P}>0.05)$; at the other three time points ( $24 \mathrm{~h}, 72 \mathrm{~h}$ and $168 \mathrm{~h}$ ), the CGRP level in the TBI-only group was markedly higher than that in the control group, and the serum level of CGRP increased following TBI in the TBI-only group, indicating that TBI was the only 'generator' for the increase of CGRP in serum in this group. The brain is protected as a priority following TBI as the result of stress response. CGRP is one of the most essential molecules in this stress protection (22). The peripheral CGRP concentrates in the brain tissue due to the disruption of the blood-brain barrier following TBI. However, the period this stress protection is brief and lasts for no longer than $48 \mathrm{~h}$. We propose that the lower CGRP levels in the serum at $48 \mathrm{~h}$ in the TBI-fracture and TBI-only groups were due to this stress response. This hypothesis requires extensive experimental demonstration and we are carrying out related experiments. Taken together, these data indicate that CGRP expression contributes significantly to an increase in fracture healing.

The putative accelerated osteogenic effects of fracture in the TBI-fracture group are thought to result from the release of CGRP from injured neural tissue or as part of a central nervous system response to brain injury. Our studies have shown that CGRP is expressed extensively in the cortex and that expression markedly increases around the fracture site in the TBI-fracture group but not in the fracture-only group. Therefore, overexpression of CGRP can only be attributed to TBI. Notably, an association between the overexpression of CGRP in the brain and around the fracture site has been observed. The mechanism by which CGRP is secreted by the injured brain and transported to the fracture site is the main pathway of accelerated fracture-healing. In this context, our studies have shown that the dynamic changes in CGRP levels in serum are similar to the changes detected around fractured femurs. Our results support the hypothesis that CGRP is the main factor transported through the serum for TBI-induced rapid bone-healing. We are the first group to define this mechanism, including the role of the neuropeptide CGRP and the mechanism by which it operates.

This area of research, which overlaps neuroscience and orthopedics, is complex and exciting. From a fundamental point of view, understanding the sophisticated mechanism involved in accelerated fracture-healing is still in its early stages, and much more remains to be revealed regarding the method by which a complex series of signals from hormones, growth factors, mechanical and neuronal changes leads to this clinical phenomenon. The exact mechanism by which the central nervous system controls bone remodeling is of great interest and presents a challenge. The hypothalamus is generally accepted as the endocrine organ, but we have revealed that other parts of the brain are also involved in the control of bone mass. Future efforts focusing on a therapeutic point of view, identifying neuronal signaling systems may be helpful in generating new bone anabolic drugs. Although CGRP is one of the unidentified humoral factors that causes enhanced osteogenesis in patients with TBI, studies regarding CGRP are likely to aid in understanding the mechanism behind fracture-healing following TBI, thereby allowing for rapid bone-healing.

\section{Acknowledgements}

This study was supported by the NSF of China (Grant no. 30872634 and no. 30901532 ).

\section{References}

1. Perkins R and Skirving AP: Callus formation and the rate of healing of femoral fractures in patients with head injuries. J Bone Joint Surg Br 69: 521-524, 1987.

2. Bidner SM, Rubins IM, Desjardins JV, et al: Evidence for a humoral mechanism for enhanced osteogenesis after head injury. J Bone Joint Surg Am 72: 1144-1149, 1990.

3. Shih $\mathrm{C}$ and Bernard GW: Calcitonin gene related peptide enhances bone colony development in vitro. Clin Orthop Relat Res 334: 335-344, 1997.

4. Valentijn K, Gutow AP, Troiano N, et al: Effects of calcitonin gene-related peptide on bone turnover in ovariectomized rats. Bone 21: 269-274, 1997.

5. Gangula PR, Lanlua P, Wimalawansa S, et al: Regulation of calcitonin gene-related peptide expression in dorsal root ganglia of rats by female sex steroid hormones. Biol Reprod 62: 1033-1039, 2000.

6. Bonnarens F and Einhorn TA: Production of a standard closed fracture in laboratory animal bone. J Orthop Res 2: 97-101, 1984.

7. Foda MA and Marmarou A: A new model of diffuse brain injury in rats. Part II: morphological characterization. J Neurosurg 80: 301-313, 1994.

8. Marmarou A, Foda MA, van den Brink W, et al: A new model of diffuse brain injury in rats. Part I: pathophysiology and biomechanics. J Neurosurg 80: 291-300, 1994

9. Arrieta-Cruz I, Pfaff DW and Shelley DN: Mouse model of diffuse brain damage following anoxia, evaluated by a new assay of generalized arousal. Exp Neurol 205: 449-460, 2007.

10. Boes M, Kain M, Kakar S, et al: Osteogenic effects of traumatic brain injury on experimental fracture-healing. J Bone Joint Surg Am 88: 738-743, 2006.

11. Gautschi OP, Cadosch D, Frey SP, et al: Serum-mediated osteogenic effect in traumatic brain-injured patients. ANZ J Surg 79: 449-455, 2009.

12. Gautschi OP, Toffoli AM, Joesbury KA, et al: Osteoinductive effect of cerebrospinal fluid from brain-injured patients. J Neurotrauma 24: 154-162, 2007.

13. Petzold A, Keir G, Lim D, et al: Cerebrospinal fluid (CSF) and serum S100B: release and wash-out pattern. Brain Res Bull 61: 281-285, 2003

14. Klein BY, Shohami E, Reikhinshtein Y, et al: Serum-mediated osteogenic effects of head injury on cultured rat marrow stromal cells. Calcif Tissue Int 65: 217-222, 1999.

15. Banerjee S, Evanson J, Harris E, et al: Identification of specific calcitonin-like receptor residues important for calcitonin generelated peptide high affinity binding. BMC Pharmacol 6: 9, 2006. 
16. Huebner AK, Keller J, Catala-Lehnen $\mathrm{P}$, et al: The role of calcitonin and alpha-calcitonin gene-related peptide in bone formation. Arch Biochem Biophys 473: 210-217, 2008.

17. Jones KB, Mollano AV, Morcuende JA, et al: Bone and brain: a review of neural, hormonal, and musculoskeletal connections Iowa Orthop J 24: 123-132, 2004.

18. Vignery A and McCarthy TL: The neuropeptide calcitonin gene-related peptide stimulates insulin-like growth factor I production by primary fetal rat osteoblasts. Bone 18: 331-335, 1996.

19. Harzenetter MD, Novotny AR, Gais P, et al: Negative regulation of TLR responses by the neuropeptide CGRP is mediated by the transcriptional repressor ICER. J Immunol 179: 607-615, 2007.
20. Tanaka S and Nakano H: NF-kappaB2 (p100) limits TNF-alpha-induced osteoclastogenesis. J Clin Invest 119: 2879-2881, 2009.

21. Imai S and Matsusue Y: Neuronal regulation of bone metabolism and anabolism: calcitonin gene-related peptide-, substance $\mathrm{P}_{-}$, and tyrosine hydroxylase-containing nerves and the bone. Microsc Res Tech 58: 61-69, 2002.

22. Holland JP, Sydserff SG, Taylor WA, et al: Calcitonin gene-related peptide reduces brain injury in a rat model of focal cerebral ischemia. Stroke 5: 2055-2059, 1994. 